
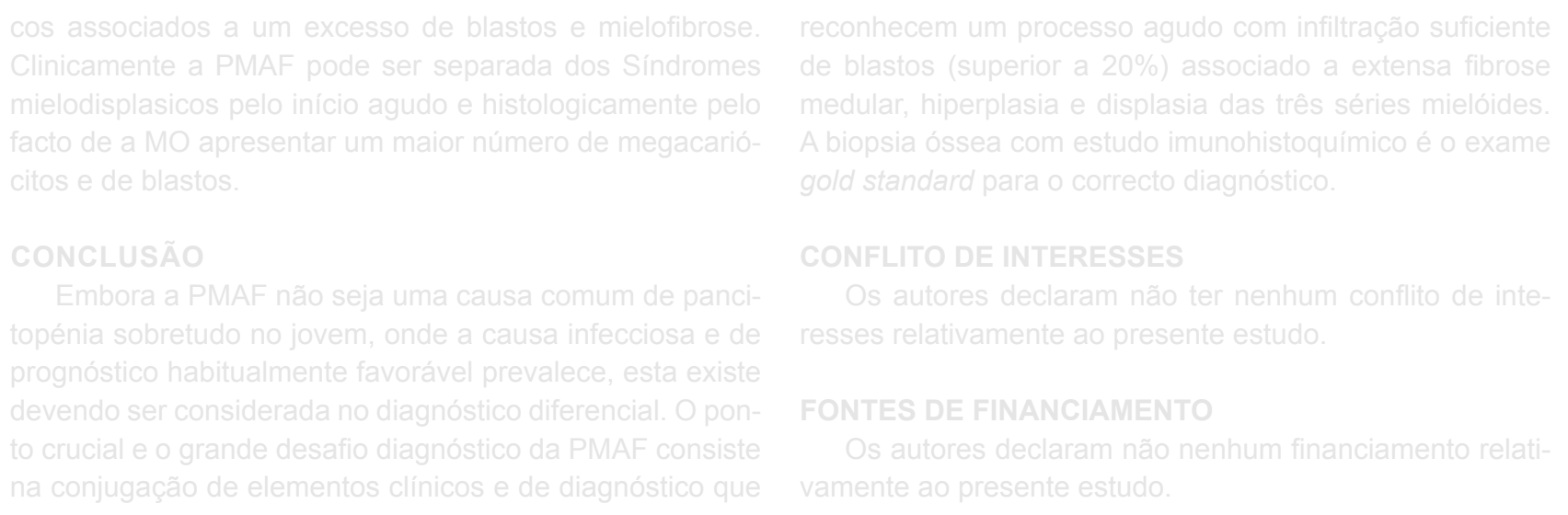

FONTES DE FINANCIAMENTO
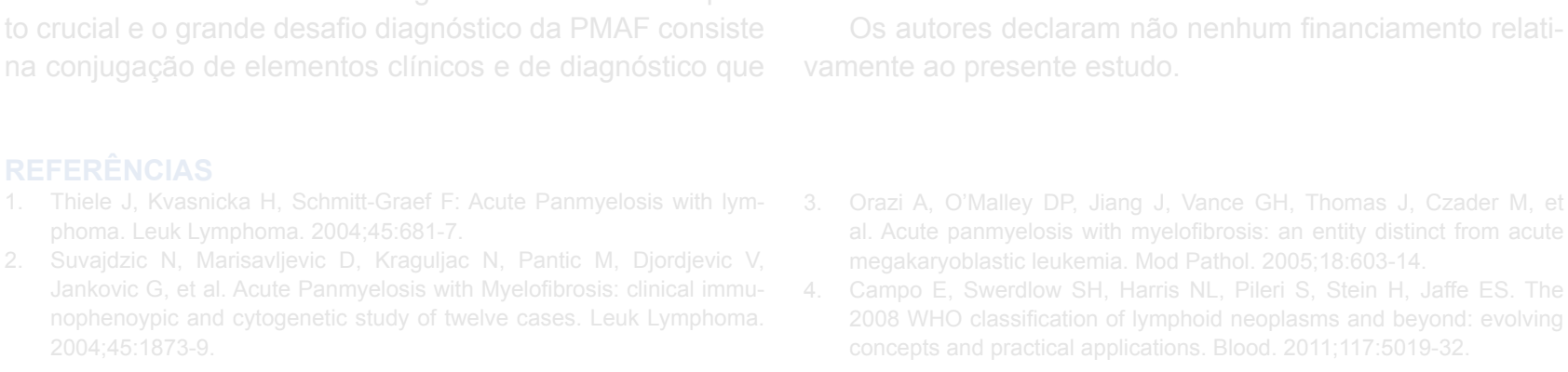

\title{
Obstrução Congénita dos Canais Ejaculadores: Diagnóstico e Resolução em Dois Casos de Sucesso
}

\section{Congenital Obstruction of the Ejaculatory Ducts: Diagnosis and Resolution of Two Successful Cases}

\author{
Rui AMORIM ${ }^{1}$, Vitor OLIVEIRA ${ }^{1}$, Jorge DIAS ${ }^{1}$, Rui FREITAS ${ }^{2}$, Luis FERRAZ1 \\ Acta Med Port 2013 Sep-Oct;26(5):616-620
}

\section{RESUMO}

A obstrução dos canais ejaculadores é um achado infrequente no estudo do factor masculino em casais inférteis. Apresentamos dois casos referenciados à nossa consulta por infertilidade conjugal primária, com factor masculino comprovado (ejaculado de baixo volume e azoospermia). Ao exame objectivo masculino não havia alterações em ambos os pacientes. Os exames de imagem demonstraram estrutura quística intra-prostática, condicionando dilatação dos canais ejaculadores. Foram submetidos a ressecção transuretral dos canais ejaculadores. O espermograma 3 meses pós-operatório demonstrou 1 caso de normalização e 1 caso de melhoria dos parâmetros espermáticos. O casal 1 tem 2 filhos, sem necessidade de técnicas de reprodução medicamente assistida. 0 casal 2 apresentou gravidez após Injecção Intracitoplasmática de Espermatozóides do ejaculado. Este diagnóstico depende de uma suspeita clínica forte e é de elevada importância, pois esta patologia tem tratamento cirúrgico eficaz. Assim, os autores pretendem realçar a relevância da avaliação andrológica aquando do estudo da infertilidade conjugal.

Palavras-chave: Canais Ejaculadores/anomalias congénitas; Doenças dos Orgãos Genitais Masculinos; Infertilidade Masculina.

\section{ABSTRACT}

The ejaculatory ducts obstruction is an uncommon finding in the study of infertility male factor. We present two cases referred to our department for conjugal infertility, with proven male factor (low ejaculate volume and azoospermia). On physical examination there was no abnormality in both patients. Imaging studies demonstrated intra-prostatic cystic structure, causing ejaculatory ducts dilation. We performed transurethral resection of the ejaculatory ducts. Semen analysis 3 months postoperatively showed 1 case of normalization and 1 case of improvement in sperm count parameters. The couple 1 couple has 2 children, without medical assisted reproductive techniques. The couple 2 had one pregnancy after Intracytoplasmic Sperm Injection, from the ejaculate. This diagnosis depends on a strong clinical suspicion and is of high importance, since this disease has effective surgical treatment. The authors aim to highlight the relevance of the assessment andrological when studying marital infertility.

Keywords: Ejaculatory Ducts/abnormalities; Genital Diseases, Male/diagnosis; Infertility, Male. 


\section{INTRODUÇÃO}

A obstrução dos canais ejaculadores (OCE) é responsável por cerca de $5 \%$ dos casos de infertilidade masculina. A OCE pode ser congénita ou adquirida. As causas congénitas incluem atresia, estenose ou quisto dos canais ejaculadores, quisto do canal de Müller (incluindo utrículo prostático) ou quisto de estruturas resultantes do canal de Wolff (Fig. 1). As causas adquiridas podem ser secundárias a traumatismo, iatrogenia, alterações infecciosas/inflamatórias, quistos prostáticos de retenção ou hiperplasia benigna da próstata com degeneração quística (HBP).

Os canais ejaculadores têm origem embriológica na mesoderme, correspondendo bilateralmente à porção terminal do Canal de Wolff ou mesonefro. Na sua anatomia definitiva são 2 canais simétricos, correspondendo à junção da vesícula seminal e canal deferente, à direita e à esquerda. Cada canal tem cerca de $2 \mathrm{~cm}$ de trajecto intra-prostático, com abertura na uretra prostática ao nível do verumontanum, lateralmente ao utrículo prostático. Este último é remanescente embriológico do canal de Müller e não apresenta comunicação directa com qualquer outra das estruturas descritas.

A sintomatologia associada a esta patologia é variada e não patognomónica. Inclui hipospermia, diminuição da força do ejaculado, hemospermia, dor ejaculatória, de localização perineal ou escrotal. A história clínica dirigida poderá revelar antecedentes de orqui-epididimite, prostatite e/ou infertilidade. $O$ exame objectivo é normalmente pobre em achados patológicos sendo de vital importância a exclusão de ausência dos canais deferentes.

Os exames complementares de diagnóstico iniciais incluem o espermograma e estudo hormonal sumário, com doseamento da FSH. O espermograma apresenta baixo volume do ejaculado ( $\mathrm{vol}<1,5 \mathrm{~mL}$ ), azoospermia ou oligozoospermia, $\mathrm{pH}$ ácido e ausência de frutose. $\mathrm{O}$ doseamento hormonal é tipicamente normal. Após suspeita clínica e analítica de patologia obstrutiva dos ejaculadores a ecografia transrectal (ETR) poderá demonstrar a presença de alterações inflamatórias peri-ejaculadores ou estrutura quística intra ou peri-prostática, medial ou lateral em relação com os mesmos (Tabela 1). Outros exames de imagem, como a tomografia computorizada (TC) e, principalmente, a ressonância magnética nuclear (RMN) poderão dar mais informações acerca da estrutura quística e sua relação com as estruturas adjacentes. A deferente-vesiculografia deverá ser utilizada em casos particulares de indefinição do nível de obstrução e poderá ser realizada intra-operatoriamente como auxiliar na decisão do tratamento cirúrgico.

A terapêutica cirúrgica está indicada, com a punção/esclerose de estruturas quísticas laterais ou a ressecção transuretral dos canais ejaculadores (TransUrethral Ressection of the Ejaculatory Ducts - TURED) no caso das estruturas quísticas mediais e obstrução intrínseca dos canais ejaculadores. Nas estruturas quísticas comunicantes com a via seminal a punção directa, por via transrectal, poderá permitir a recolha de espermatozóides viáveis para criopreservação.

A resolução da obstrução é conseguida em mais de $50 \%$ dos casos, estando descritos diferentes resultados de acordo com a origem etiológica da obstrução.

\section{CASO CLÍNICO}

Os autores apresentam 2 casos de infertilidade conjugal primária com duração superior a 24 meses, sem outros antecedentes patológicos de relevo. Em ambos os casos o espermograma revelou hipospermia, pH baixo, ausência de frutose e azoospermia.

No caso 1 a ETR demonstrou quisto intraprostático, próxima dos canais ejaculadores. A RMN demonstrou compressão extrínseca dos mesmos (Fig. 2). No caso 2 a ETR demonstrou quisto intraprostático da linha média, com dilatação das vesículas seminais (Fig. 3).

Em ambos os casos realizou-se intra-operatoriamente deferente-vesiculografia, que comprovou obstrução ao nível dos canais ejaculadores. No caso 2 houve preenchimento por contraste do quisto e refluxo para a vesícula seminal contralateral (Fig. 4). Neste caso a punção transrectal do quisto permitiu a recolha de espermatozóides. No caso 1 a punção da vesícula seminal permitiu a recolha

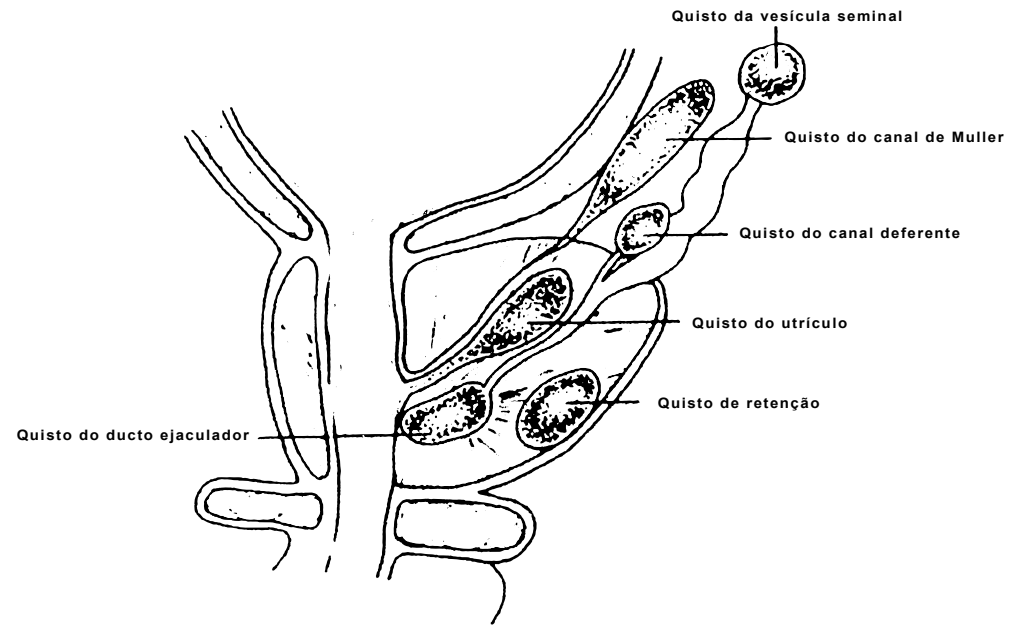

Figura 1 - Diagrama das lesões císticas intra e peri-prostáticas (adaptado de 'Cystic Lesions of the Prostate'). 

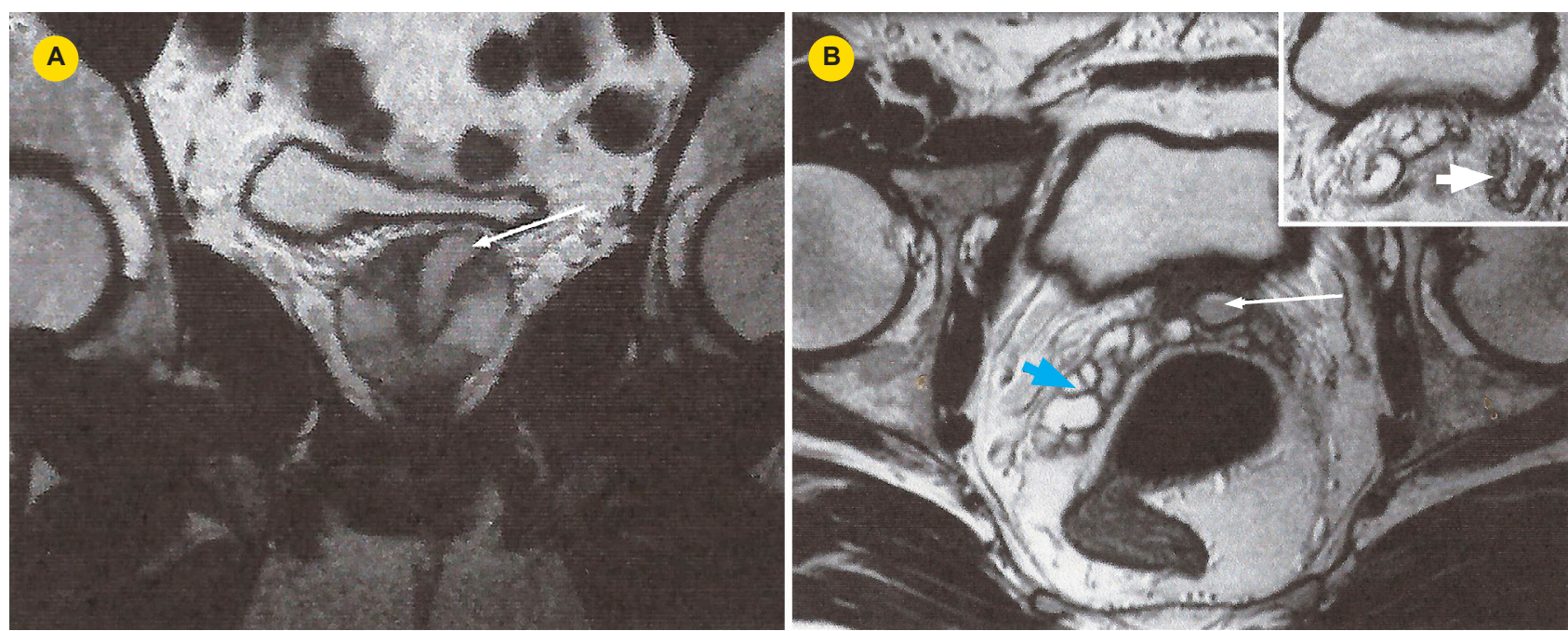

Figura 2 - RMN: A- quisto prostático paramediano; B- quisto prostático (seta branca fina); vesícula seminal direita dilatada (seta azul); vesícula seminal esquerda não dilatada (canto superior direito).

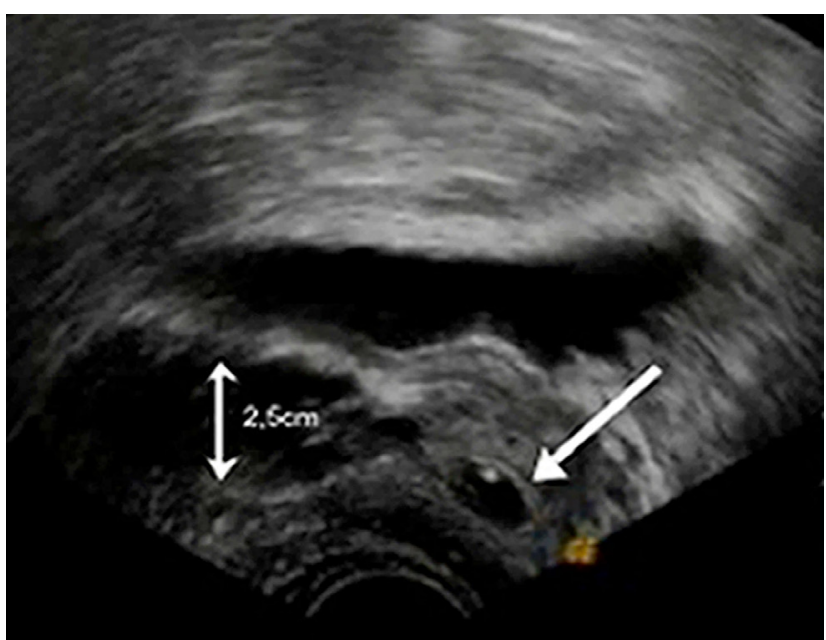

Figura 3 - Ecografia do caso 2 evidenciando lesão cística (seta branca) e dilatação da vesícula seminal $(2,5 \mathrm{~cm})$.

de milhões de espermatozóides mas a punção do quisto demonstrou ausência de espermatozóides.

Após injecção de azul de metileno por punção directa da vesícula seminal, realizou-se a TURED, constatando-se imediata ejecção abundante de material viscoso, com azul de metileno (Fig. 5).

No caso 1 houve normalização da totalidade dos parâmetros do espermograma aos 3 meses. Actualmente com dois filhos, saudáveis, sem recurso a técnicas de fertilização.

No caso 2 houve normalização do volume do ejaculado $(5 \mathrm{~mL})$ e do valor da frutose $(250 \mathrm{mg} / \mathrm{dL})$, com espermatozóides presentes mas com necrozoospermia associada. Foi obtida gravidez após injecção intracitoplasmática de espermatozóides (Intra Cytoplasmic Sperm Injection - ICSI), com espermatozóides recolhidos a partir do ejaculado.

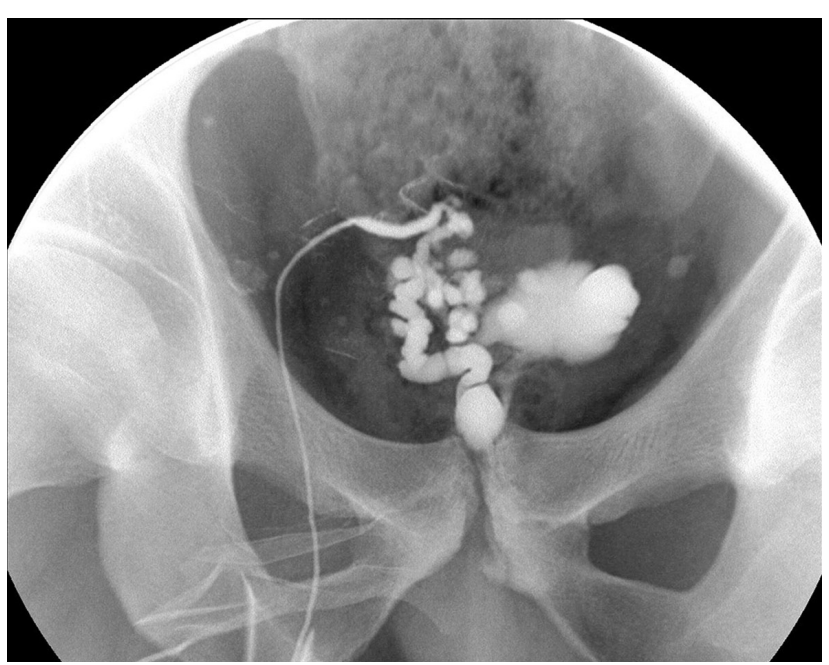

Figura 4 - Deferente-vesiculografia do caso 2 evidenciando preenchimento da estrutura quística, obstrução terminal do canal ejaculador direito com refluxo contralateral.

\section{DISCUSSÃO}

Durante o estudo do factor masculino de infertilidade, quando existem alterações do espermograma compatíveis com obstrução dos canais ejaculadores (hipospermia - excluídos os casos de má técnica/condições de colheita -, $\mathrm{pH}$ ácido, azoospermia e frutose baixa) deve ser realizada ETR. A identificação de estrutura quística intra-prostática indicia alterações do desenvolvimento embriológico, podendo representar quisto do canal de Müller ou do canal de Wolff (incluindo vesículas seminais e canais ejaculadores), ou alterações adquiridas como quisto de retenção prostático.

No caso 1 a observação de quisto intra-prostático comprimindo extrinsecamente os canais ejaculadores, sem comunicação com estes e sem espermatozóides no seu interior é a favor de quisto de Müller ou quisto do utrículo, pois estas estruturas têm origem embriológica diferente 


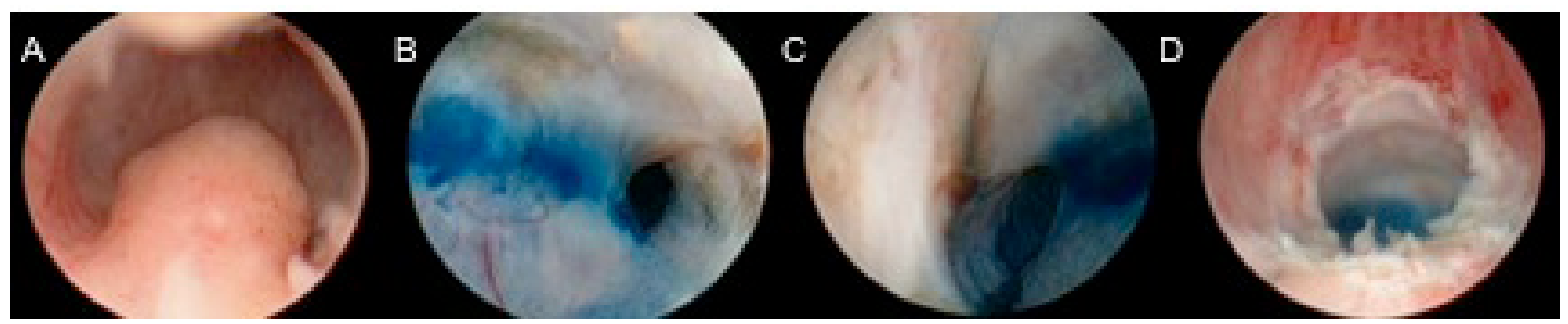

Figura 5 - Imagem de cistoscopia evidenciando quisto da linha média (A); inserção dos ductos ejaculadores (B, C); resultado final após TURED (D).

Tabela 1 - Diagnóstico diferencial de lesões prostáticas

\section{Lesões da linha média}

Lesões laterais
Quisto do utrículo

Quisto do Canal de Mullër

Quisto do ducto ejaculador

Quisto do canal deferente
Quisto de retenção

HBP (degeneração quística)

Abcesso

Quisto da vesícula seminal

\section{Sem espermatozóides}

Com espermatozóides

Sem espermatozóides

Quisto de retenção

Quisto do ducto ejaculador

Quisto do canal deferente
Quisto do utrículo

Quisto do Canal de Müller
Quisto da vesícula seminal
HBP (degeneração quística)

Abcesso

das estruturas genitais masculinas.

No caso 2 a identificação de quisto intra-prostático que comunica com ambos os canais ejaculadores, contendo espermatozóides, está de acordo com quisto do canal de Wolff.

Em ambos os casos a TURED resolveu o processo obstrutivo e permitiu a melhoria/normalização dos parâmetros do espermograma. Este tratamento permitiu a gravidez espontânea ou a recolha de espermatozóides a partir do ejaculado para técnicas de fertilização. Para além disso, o aumento do volume do esperma representa para estes doentes uma nova e agradável realidade na sua função ejaculatória.

Tal como nos 2 casos descritos, a azoospermia associada a hipospermia, $\mathrm{pH}$ ácido e ausência de frutose no ejaculado indicam patologia obstrutiva dos canais ejacula- dores, que pode ter cura cirúrgica definitiva.

Fica assim demonstrada a necessidade do estudo do factor masculino ser efectuado por um Urologista com experiência na área da Infertilidade, com elevado nível de conhecimentos em Embriologia, de forma a identificar não só as patologias mais frequentes mas também as mais raras e capaz de orientar rapidamente o casal para o tratamento mais eficaz.

\section{CONFLITO DE INTERESSES}

Os autores declaram não ter nenhum conflito de interesses relativamente ao presente estudo.

\section{FONTES DE FINANCIAMENTO}

Os autores declaram não nenhum financiamento relativamente ao presente estudo.

\section{REFERÊNCIAS}

1. Fisch H, Kang YM, Johnson CW, Goluboff ET. Ejaculatory duct obstruction. Curr Opin Urol. 2002;12:509-15.

2. Popken G, Wetterauer U, Schultze-Seemann W, Deckart A, Sommerkamp $\mathrm{H}$. Transurethral resection of cystic and non-cystic ejaculatory

duct obstructions. Int J Androl. 1998;21:196-200.

3. Johnson CW, Bingham J, Goluboff E, Fisch H. Transurethral resection of the ejaculatory ducts for treating ejaculatory symptoms. New York: College of Physicians and Surgeons of Columbia University; 2004.

4. Turek PJ, Magana JO, Lipshultz LI. Semen parameters before and 
after transurethral surgery for ejaculatory duct obstruction. J Urol. 1996;155:1291-3.

5. Pryor JP, Hendry WF. Ejaculatory duct obstruction in subfertile males: analysis of 87 patients. Fertil Steril. 1991;56:725-30.

6. Goldwasser BZ, Weinerth JL, Carson CC 3rd. Ejaculatory duct obstruction: the case for aggressive diagnosis and treatment. J Urol.1985;134:964-6.

7. Goluboff ET, Stifelman MD, Fisch $\mathrm{H}$. Ejaculatory duct obstruction in the infertile male. Urology. 1995;45:925-31.

8. Kuligowska E, Baker CE, Oates RD. Male infertility: role of transrectal US in diagnosis and management. Radiology. 1992;185:353-60.

9. Cornud F, Belin X, Delafontaine D, Amar T, Hélénon O, Moreau JF. Imaging of obstructive azoospermia. Eur Radiol. 1997;7:1079-85.

10. Meacham RB, Hellerstein DK, Lipshultz LI. Evaluation and treatment of ejaculatory duct obstruction in the infertile male. Fertil Steril. 1993;59:393-7.

11. Jarow JP. Transrectal ultrasonography of infertile men. Fertil Steril.
1993:60:1035-9.

12. Littrup PJ, Lee F, McLeary RD, Wu D, Lee A, Kumasaka GH. Transrectal US of the seminal vesicles and ejaculatory ducts: clinical correlation. Radiology.1988;168:625-8.

13. Farley S, Barnes R. Stenosis of ejaculatory ducts treated by endoscopic resection. J Urol. 1973;109:664-6.

14. Goluboff ET, Kaplan SA, Fisch H. Seminal vesicle urinary reflux as a complication of transurethral resection of the ejaculatory ducts. J Urol. 1995;153:1234-5.

15. Pryor JP, Schlegel PN. Management of ejaculatory duct obstruction. In Lipshultz LI, Howard SS, editors. Infertility in the Male. $3^{\text {rd }}$ ed. Chapter 21. St. Louis: Mosby; 1997. p.187.

16. Jarow JP, Espeland MA, Lipshultz LI. Evaluation of the azoospermic patient. J Urol.1989;142:62-5.

17. Nagler HM, Rotman M, Zoltan E, Fisch $\mathrm{H}$. The natural history of partial ejaculatory duct obstruction. J Urol. 2002;167:253-4. 